\title{
Towards an Improved Ensemble Precipitation Forecast: A Probabilistic Post-Processing Approach
}

\author{
Sepideh Khajehei and Hamid Moradkhani
}

\begin{abstract}
Recently, ensemble post-processing (EPP) has become a commonly used approach for reducing the uncertainty in forcing data and hence hydrologic simulation. The procedure was introduced to build ensemble precipitation forecasts based on the statistical relationship between observations and forecasts. More specifically, the approach relies on a transfer function that is developed based on a bivariate joint distribution between the observations and the simulations in the historical period. The transfer function is used to post-process the forecast. In this study, we propose a Bayesian EPP approach based on copula functions (COP-EPP) to improve the reliability of the precipitation ensemble forecast. Evaluation of the copula-based method is carried out by comparing the performance of the generated ensemble precipitation with the outputs from an existing procedure, i.e. mixed type meta-Gaussian distribution. Monthly precipitation from Climate Forecast System Reanalysis (CFS) and gridded observation from Parameter-Elevation Relationships on Independent Slopes Model (PRISM) have been employed to generate the post-processed ensemble precipitation. Deterministic and probabilistic verification frameworks are utilized in order to evaluate the outputs from the proposed technique. Distribution of seasonal precipitation for the generated ensemble from the copula-based technique is compared to the observation and raw forecasts for three sub-basins located in the
\end{abstract}


25 Western United States. Results show that both techniques are successful in producing reliable

26 and unbiased ensemble forecast, however, the COP-EPP demonstrates considerable

27 improvement in the ensemble forecast in both deterministic and probabilistic verification, in

28 particular in characterizing the extreme events in wet seasons.

29 Post-Processing; Precipitation; Copulas; Climate Forecast System; Hydrologic Forecasting

\section{Introduction}

31 Uncertainty in hydrologic simulation and forecast arises from the uncertainties associated with

32 the forcing data, parameters, initial condition, and hydrologic model structure. To achieve

33 accurate hydrologic forecasts, each of these components should be estimated accurately. In the

34 past few years, researchers have proposed various techniques to tackle uncertainty in hydrologic

35 modeling from different angles. For instance, data assimilation is often proposed to deal with the uncertainty in the initial and boundary conditions ( DeChant and Moradkhani, 2014; Li et al.,

37 2009; Zehe and Blöschl, 2004). The skill of forecasts can be enhanced by post-processing 38 through multi-modeling (Duan et al., 2007; Madadgar and Moradkhani, 2014a; ; Najafi and 39 Moradkhani, 2015), or other statistical methods, such as quantile mapping or non-parametric 40 procedures (Madadgar et al., 2014; Wood and Schaake, 2008; Zhao et al., 2011; Zhao et al., 41 2015); Generalized Linear Model Post-Processor (GLMPP) (Ye et al., 2014; Zhao et al., 2011); 42 and the combination of data assimilation and post processing (Bourgin et al., 2014). In addition, 43 characterizing uncertainty in forcing data has received attention in recent years (Clark and Vrugt, 44 2006; Kavetski et al., 2006; Raleigh et al., 2015; Steinschneider et al., 2012). Moreover, 45 techniques have been developed for generating ensemble meteorological forecasts (Clark et al., 46 2004; Clark and Hay, 2004; Robertson et al., 2013; Schaake et al., 2007; Tao et al., 2014; Wu et 
47 al., 2011; Zhao et al., 2011). Ensemble precipitation forecasts provide the forecasts of the most

48 likely events as well as the uncertainty information. (Park et al., 2008; Tao et al., 2014).

49 Products from the National Centers for Environmental Prediction (NCEP) models are available 50 in a diverse range of spatial and temporal scales. For instance, Short Range Forecast System 51 (SREF), Global Ensemble Forecast System (GEFS), and Climate Forecast System (CFS) are 52 widely utilized in various studies (Kumar et al., 2012; Peng et al., 2013; Wang et al., 2014). 53 Despite all of these developments in climate models, forecasts are still prone to bias in the mean 54 and insufficient spread (Hamill and Whitaker, 2006; Hamill et al., 2006; Robertson et al., 2013; 55 Tao et al., 2014; Wu et al., 2011). These errors are more significant in climate variables, such as 56 precipitation, which are affected immensely by changes in spatial scale. Thus, it is recommended 57 to not employ the ensemble products from climate models directly, due to three main reasons: 1) 58 there is low accuracy in the ensemble climate forecasts, 2) The models are developed with 59 various assumptions which may not necessarily hold for every regions, and 3) models are mostly 60 developed for large scale applications, and therefore, even if the variables are free from error at 61 their original scale, they might be biased at the catchment scale (Rayner et al., 2005; Tao et al., 62 2014; Wu et al., 2011).

63 There is a demand for methods that are able to generate reliable ensemble forecasts for 64 hydrologic applications. A promising approach is to apply statistical procedures to generate 65 ensemble forecast from Numerical Weather Prediction (NWP) -generated single-value forecasts.

66 The procedure is based on the bivariate probability distribution between the observation and the 67 single-value precipitation forecast. In the past few years, various methods were applied to meet 68 this objective. Kelly and Krzysztofowicz (1997) developed a bivariate meta-Gaussian 69 distribution function based on a normal quantile transformation of two variables according to the 
70 Gaussian law in the Bayesian Forecasting System (BFS). The method was later used by

71 Krzysztofowicz and Herr (2001) to assess the uncertainty in the precipitation data. Clark and

72 Hay (2004) employed Model Output Statistics (MOS) to downscale the model outputs of Global

73 Forecast System (GFS), a medium range forecast system, developed in the National Weather

74 Service (NWS) cooperative network. To preserve and represent space-time variability of climate

75 variables, Clark et al. (2004) introduced a procedure, the so called Schaake Shuffle

76 reconstructing the ensemble members according to the historical values. Schaake et al. (2007)

77 described a full procedure, which is used at the National Weather Service River Forecasting

78 System (NWSRFS) for developing the ensemble meteorological forecasts as the input to the

79 ESP. It is based on the mixed distribution of the two variables and applying the normal quantile

80 transformation for building the joint distribution between the two non-normal variables. In

81 addition to the above mentioned methods, Linear Regression methods have been employed in

82 various studies to post-process precipitation at different temporal and spatial scales (Roulin \&

83 Vannitsem, 2012; Sumner, Homar, \& Ramis, 2001; Wilks, 2009).

84 Recently, Wu et al. (2011) developed the Mixed type meta-Gaussian joint distribution built upon

85 the method of Kelly and Krzysztofowicz (1997), which models the precipitation intermittency

86 decisively in comparison to the Schaake et al. (2007) method, which models each of the marginal

87 distributions as a convex combination of the continuous distributions. Robertson et al. (2013)

88 used the Bayesian Joint Probability approach (BJP) developed by Wang and Robertson (2011),

89 and Wang et al. (2009) for generating the ensemble precipitation forecast for a sub-daily weather

90 forecast in Australia. The number of parameters is one of the challenges in EPP; therefore, one of

91 the merits of BJP is that it uses a lesser number of parameters. Tao et al. (2014) employed EPP in

92 combination with multi-modeling to generate a more reliable forecast from The Observing 
93 System Research and Predictability Experiment (THORPEX) Interactive Grand Global

94 Ensemble (TIGGE) products.

95 According to the above methods, it is assumed that the joint probability distribution between the 96 observations and the forecasts is following a multivariate normal distribution. Furthermore, using 97 normal joint distribution leads to the necessity for a transformation of the non-Gaussian variable 98 into the normal space. The transformation may affect the accuracy of the estimated probability 99 distribution (Brown and Seo, 2013; Madadgar and Moradkhani, 2014a). Therefore, Brown and 100 Seo (2013) introduced a nonparametric probability distribution. Also, Madadgar et al. (2014) 101 demonstrated that the nonparametric probability distributions are highly dependent on the 102 number of thresholds for observed variables. Consequently, it is beneficial to develop a 103 procedure that can capture the uncertainty in a way that there would be no need for variable 104 transformation, and simultaneously adopt a true probability distribution between observation and 105 forecast. Sklar (1959) introduced the concept of constructing multivariate distributions using 106 copulas. Copula functions are useful in capturing the dependency in most of the multivariate 107 distributions such as, bivariate Pareto and bivariate gamma. Copula functions have the capability 108 to draw the joint distribution regardless of the marginal distribution (Favre et al., 2004; Joe, 109 1997). Copula functions have been used substantially in hydrological applications including 110 precipitation estimation and drought forecasting (Bárdossy, 2006; Bárdossy and Pegram, 2013; 111 Bárdossy and Pegram, 2014; Dung et al., 2015; Favre et al., 2004; Madadgar and Moradkhani, 112 2013; Madadgar and Moradkhani, 2014b; Salvadori and De Michele, 2010). Madadgar et al. 113 (2014) proposed copula functions to construct the joint distribution between two variables with 114 any level of dependency because they have the potential to be applicable in post-processing the 115 hydrological simulation based on the observation and modeled streamflow simulations. They 
116 showed that the copula-based method could provide a more accurate and skillful forecast in

117 comparison to the quantile mapping approach, a widely-used post-processing method.

118 In this study, we evaluate the capability of copula functions to build the joint distribution 119 between the observation and climatological forecast. This paper is organized in five sections. 120 The introduction is followed by methodology in Section 2, which includes a description of the 121 two techniques that are used in the study, as well as the forecast verification metrics that are 122 employed to evaluate the generated forecasts. Section 3 describes the data and study area. 123 Results and discussion are elaborated in Section 4, and finally, the summary and conclusion are 124 provided in Section 5.

\section{2. Methodology}

126 The main assumptions in post-processing the forecasts are that the observation and forecast are 127 correlated, and that future behavior of the system will remain the same. The purpose of this study 128 is to integrate copula functions into the EPP framework. Copula functions relax these 129 assumptions, and are able to build ensemble forecasts based on historical observations and 130 climatological forecasts. To assess the robustness and reliability of this new technique, results 131 are compared with the outputs from an existing approach called Mixed type Bivariate meta132 Gaussian distribution (MBG), introduced by Wu et al. (2011). The theory of copula functions is 133 provided below.

\subsection{Data classification and Marginal Distribution}

135 The dataset is divided into twelve monthly classes, and the procedure is applied to each class 136 separately. The procedure starts with fitting different marginal distributions to the observations 137 and forecasts. These distributions include Weibull, Exponential, Lognormal, and Gamma, which 138 are well suited to non-negative data (i.e. precipitation in this case). Furthermore, they are 
139 powerful in representing the extreme values of precipitation data. To find the best marginal 140 distribution that can describe the observation and forecast values, Bayesian Information Criterion 141 (BIC) and Kolmogorov-Smirnov (K-S) tests are employed. For a detailed description of these 142 tests, readers are referred to Aho et al. (2014), Raftery (1986), and Stephens (1974).

\subsection{Bivariate Meta-Gaussian Distribution (MBG) Approach}

144 Here we discuss generating ensembles through the MBG method (Wu et al., 2011). The goal of 145 this approach is to build the joint cdf of two variables, the observation $(\mathrm{O})$, and the forecast $(\mathrm{Y})$, 146 and then perform sampling from this cdf to generate the ensemble members at each time step.

$$
F(y, o)=P(Y \leq y, O \leq o)
$$

147 Since the joint cdf is going to be built based on the bivariate meta-Gaussian distribution, 148 probability distribution of observation and forecast requires to be transformed into normal space. 149 Normal Quantile Transform (NQT) is employed to derive $\mathrm{W}$ and $\mathrm{Z}$ as the replacements of $\mathrm{F}(\mathrm{O})$ 150 and $\mathrm{F}(\mathrm{Y})$, the cdfs of observation and forecast in the normal space, respectively.

$$
\begin{aligned}
& Z=Q^{-1}(F(Y)) \\
& W=Q^{-1}(F(O))
\end{aligned}
$$

151 Where, Q denotes the standard normal distribution function.

\section{$152 \quad$ 2.2.1. Building and Sampling from the Conditional Joint Distribution}

153 In this step, the conditional distribution between the observation and forecast is formed. By 154 replacing the $\mathrm{y}$ and $\mathrm{o}$ by $\mathrm{z}$ and $\mathrm{w}$ respectively, it is assumed that the joint distribution between 155 observation and forecast would be equal to the bivariate normal joint distribution between $\mathrm{z}$ and $156 \mathrm{w}(B(z, w ; \rho))$ :

$$
H(y, o ; \rho) \equiv B(z, w ; \rho)
$$


157 where $\mathrm{H}(\mathrm{y}, \mathrm{o} ; \rho)$ is the bivariate meta-Gaussian distribution of $\mathrm{Y}$ and $\mathrm{O}$, as introduced by Kelly 158 and Krzysztofowicz (1997), and $\rho$ is the Pearson product-moment correlation coefficient 159 between $\mathrm{Z}$ and $\mathrm{W}$. It can be assumed that:

$$
F(y, o) \approx H(y, o ; \rho)
$$

160 According to the meta- Gaussian distribution of $\mathrm{O}$ and $\mathrm{Y}$, the conditional distribution of $\mathrm{O}$ given $161 \mathrm{Y}=\mathrm{y}$ can be written as follows:

$$
H(O \mid Y=y)=Q\left(\frac{w-\rho z}{\left(1-\rho^{2}\right)^{\frac{1}{2}}}\right)
$$

162 To create the ensemble forecast at each time step, Equation (6) is being solved employing 163 stratified sampling of the observation given forecast, and therefore we have $p=H\left(O_{\text {samples }} \mid y\right)$. 164 The ensemble members will be generated by following the Equation (7) at each p-probability.

$$
\operatorname{Member}_{p}=F_{O}^{-1}\left(Q\left(\rho z+\left(1-\rho^{2}\right)^{\frac{1}{2}} Q^{-1}(p)\right)\right)
$$

\section{2.2.2. The Schaake Shuffle}

166 To represent temporal dependence, the Schaake Shuffle is used to shuffle the ensemble members 167 at each time step according to the historical observation. In this technique, the ensemble 168 members are being ranked and matched with the historical data for the same month in the past. 169 Then, the members will be reordered to follow the same order as the one for the historical data 170 (Clark et al., 2004). 


\subsection{Ensemble Post-Processing Based on Copula Functions (COP-EPP)}

\subsubsection{The Theory of Copula Functions}

173 Copula functions were introduced by Sklar (1959) as functions in the unit cube, which can link 174 multi-dimensional distributions to their one-dimensional marginals. Mathematically, the n175 dimensional copula $C$ is represented as: $C:[0,1]^{n} \rightarrow[0,1]$.

In the bivariate case, a copula satisfies the following property:

$$
C\left(u_{1}, u_{2}\right)+C\left(v_{1}, v_{2}\right)-C\left(u_{1}, v_{2}\right)-C\left(v_{1}, u_{2}\right) \geq 0 \text { if } u_{1} \geq v_{1} \text { and } u_{2} \geq v_{2}
$$

177 Where $\mathrm{u}$ and $\mathrm{v}$ are the marginal distributions of two random variables. In $\mathrm{n}$-dimensional space, 178 the original cumulative distribution can be written as:

$$
\begin{aligned}
& F\left(x_{1}, x_{2}, \ldots, x_{n}\right)=C\left[F_{X_{1}}\left(x_{1}\right), F_{X_{2}}\left(x_{2}\right), \ldots, F_{X_{n}}\left(x_{n}\right)\right]=C\left(u_{1}, u_{2}, \ldots, u_{n}\right) \\
& C\left(u_{1}, u_{2}, \ldots, u_{n}\right)=\operatorname{Pr}\left\{U_{1} \leq u_{1}, \ldots, U_{2} \leq u_{2}\right\}
\end{aligned}
$$

Based on the derivative of cumulative density function (cdf) of the copula, the probability distribution function (pdf) of copula is obtained:

$$
c\left(u_{1}, \ldots, u_{n}\right)=\frac{\partial^{n} C\left(u_{1}, \ldots, u_{n}\right)}{\partial u_{1} \ldots \partial u_{1}}
$$

The joint density function can then be written as follows:

$$
f\left(x_{1}, \ldots, x_{n}\right)=c\left(u_{1}, \ldots, u_{n}\right) \prod_{i=1}^{n} f_{X_{i}}\left(x_{i}\right)
$$

There are several types of copula functions (Nelsen, 1999). Two of these families are applicable in hydrology, Archimedean and Elliptical (Nelsen, 1999; Sklar, 1959). In this study, five copula

187 functions have been used: Gaussian and " $\mathrm{t}$ " from Elliptical, and Frank, Clayton, and Gumbel from Archimedean categories. 
189 To find the best copula function that describes the relationship between observations and 190 forecasts, we use Goodness of Fit (GoF) test statistics based on the distance between the 191 empirical and parametric copula described by Genest et al. (2009). Cramer-von Mises and

192 Kolmogorov-Smirnov statistics were used for measuring the distance (Anderson, 1962):

$$
\begin{aligned}
& S_{n}=\int_{u} \Delta C_{n}(n)^{2} d C_{n}(u) \\
& \Delta C_{n}=\sqrt{n}\left(C_{n}-C_{\theta_{n}}\right)
\end{aligned}
$$

193 Where $C_{n}$ is the empirical copula with sample size of $n$, and $C_{\theta n}$ is the theoretical copula 194 estimated for a sample size of $\mathrm{n}$. The null hypothesis assumes that the theoretical copula fits the 195 data. Therefore, the copula function with the greatest $p$-value $(p>0.05)$ and smallest $S_{n}$ is desired. 196 To calculate the $\mathrm{p}$-value, parametric bootstrap procedure with 1000 iterations and $\alpha=0.01$ is used.

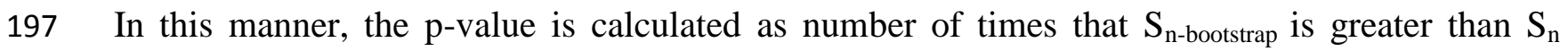
198 divided by the number of iterations. In this study, we have used Inference Functions for Margins 199 (IFM) to estimate the parameters of copula functions (Dupuis, 2007; Joe, 1997).

\subsubsection{Ensemble Construction Based on Copula Functions}

201 Ensemble members are generated by sampling from the conditional pdf of the observation given 202 forecast at each time step. In a Bayesian network, the joint distribution of observation and 203 forecast can be simplified as follows:

$$
f(y, o)=f(y) \cdot f(o \mid y)
$$

204 From Equation (15), the conditional probabilities can be derived as:

$$
f(o \mid y)=\frac{f(o, y)}{f(y)}
$$


205 By using copula functions in estimating the joint pdf (Equation (12)), the conditional pdf 206 (Equation (16)) can be decomposed to a simpler form as:

$$
f\left(s_{t} \mid y_{t}\right)=\frac{c\left(U_{s}=u_{s}, U_{Y}=u_{y}\right) f\left(y_{t}\right) f\left(s_{t}\right)}{f\left(y_{t}\right)}=c\left(U_{s}=u_{s}, U_{Y}=u_{y}\right) f\left(s_{t}\right)
$$

207 where $f\left(s_{t} \mid y_{t}\right)$ is the conditional pdf of the sample and forecast at time t, $f\left(y_{t}\right)$ and $f\left(s_{t}\right)$ are 208 the marginal pdf of the forecast, and the sample from the observation at time t. $c(.,$.$) is the$ 209 copula pdf (Madadgar et al., 2014).

210 The conditional pdf $\left(f\left(S \mid y_{t}\right)\right)$ is built by Monte Carlo sampling from the copula density 211 function $\left(c\left(U_{s}, U_{Y}=u_{y}\right)\right)$, in the following steps:1) The forecast is fixed at time $\mathrm{t}\left(U_{Y}=u_{y}\right.$ at 212 time $t$ ), 2) Generate $U_{s}$ by sampling from the historical observation (500 samples in this study). 213 3) compute the value of $c\left(U_{s}, U_{Y}=u_{y}\right)$. Now Equation (17a) is modified as follows:

$$
f\left(S \mid y_{t}\right)=c\left(U_{s}, U_{Y}=u_{y}\right) f(s)
$$

214 Following Schaake et al. (2007), to sample from the conditional pdf and to generate the 215 ensemble of size $n$ (in this study, 20) the probabilities at equal intervals $(0.05,0.1,0.15, \ldots, 0.95)$ 216 are considered. Then, the ensemble members associated with above probabilities are obtained. 217 To preserve the temporal variability, the Schaake Shuffle is used to shuffle the ensemble 218 members at each time step according to the historical observation. Figure 1 demonstrates the 219 schematic of COP-EPP procedure.

220 Figure 1. Schematic of the Copula-based ensemble post-processing (COP-EPP). The ensemble 221 members are generated by sampling from conditional pdf and reconstructed according to 222 Schaake shuffle. 


\subsection{Forecast Verification}

224 To examine the robustness, reliability, and effectiveness of the proposed approach, results of 225 both approaches, i.e. MBG and COP-EPP are compared through deterministic and probabilistic 226 verification metrics. Accordingly, one deterministic and two probabilistic measures are chosen to

227 evaluate the two generated ensembles. To have a clear comparison between results, normalized 228 measures are employed. For sufficient examination of results, the generated ensembles are 229 analyzed during both the calibration and verification periods.

\section{$230 \quad$ 2.4.1. Deterministic Measures}

231 To inspect the errors in the mean ensemble precipitation forecast, deterministic measures are 232 used. Also, it would be beneficial to study the relationship between the observation and raw 233 forecast in a deterministic framework.

234 Absolute Percent bias evaluates the deviation of the ensemble mean from the observation. The 235 optimal value of Absolute percent bias is zero (Gupta, Sorooshian, \& Yapo, 1999; Moriasi et al., 236 2007).

$$
\text { Absolute Percent Bias }=\frac{\sum_{i=1}^{n}\left|y_{i}-o_{i}\right|}{\sum_{i=1}^{n}\left(o_{i}\right)} \times 100
$$

237 To assess the accuracy of the forecast versus observation, the Kling-Gupta Efficiency (KGE) 238 measure is utilized. KGE was introduced as the modified version of Nash-Sutcliffe Efficiency 239 (NSE) by Gupta et al. (2009). This measure captures the correlation, bias, and variability in the 240 forecast data versus the observation.

$$
\begin{aligned}
& K G E=1-E D \\
& E D=\sqrt{(r-1)^{2}-(\alpha-1)^{2}-(\beta-1)^{2}}
\end{aligned}
$$


241 ED is defined as the Euclidean distance between two variables, $r$ represents the linear correlation

242 coefficient between the observation and forecast, $\alpha$ is the ratio of variance of forecast to variance

243 in observation (relative variability in the forecast and observation), and $\beta$ represents the ratio

244 bias. ED is always non-negative, and thus KGE will have a value from $-\infty$ to 1, with optimal

245 value of 1.

\section{2.4.2. Probabilistic Measures}

247 Since the deterministic measures are affected by over or under -confident forecasts, it is essential

248 to examine the generated forecast through probabilistic measures as well. Probabilistic measures

249 are beneficial in assessing the reliability of ensemble forecast (DeChant and Moradkhani, 2014b;

250 DeChant and Moradkhani, 2015). To assess the forecast skill of the generated ensembles,

251 Continuous Ranked Probability Skill Score (CRPSS) is employed. This measure is the

252 normalized version of Continuous Ranked Probability Score (CRPS) that has been introduced as

253 the extension of Brier Score over all the possible thresholds (Hersbach, 2000).

$$
\text { CRPS }=\int_{x=-\infty}^{x=\infty}\left(F^{y}(x)-F_{o}(x)\right)^{2} d x
$$

254 and

$$
F_{O}(x)=\left\{\begin{array}{l}
0, x<\text { observed value } \\
1, x \geq \text { observed value }
\end{array}\right.
$$

255 Where $F^{f o}(x)$ is the forecast probability, i.e. cdf of the forecast. In the deterministic case CRPS 256 will be the same as MAE with the optimal value of zero.

$$
C R P S S=\frac{\overline{C R P S_{\text {ref }}}-\overline{C R P S_{\text {forecast }}}}{\overline{C R P S_{\text {ref }}}}
$$


257 CRPS $_{\text {ref }}$ refers to the Continues Ranked Probability Score for the observation. The range for 258 CRPSS is $-\infty$ to one with optimal value of one.

$$
C R P S_{\text {ref }}=\frac{1}{T} \sum_{t=1}^{T} \sum|o(t)-\bar{o}|
$$

260 One of the techniques used to evaluate the forecast skill is to employ the Relative (or Receiver) 261 Operating Characteristic (ROC) curve in which the hit rate and false- alarm are compared 262 (Mason, 1982). The area under the curve represents the ROC score ranging between 0 and 1 with 263 the perfect score of 1. In this study, the ROC score is used to examine the resolution of the 264 generated forecast for winter precipitation (December, January, and February) during the 265 verification period (2000-2014).

266 The ROC score is not sensitive to bias in the forecast (Wilks, 2011); therefore, it can be 267 considered as a measure for assessing the potential usefulness of a certain forecast. On the 268 contrary, bias can affect the reliability of forecast (Murphy, 1993). Accordingly, the reliability of 269 winter precipitation is evaluated based on the decomposition of the Brier score (Murphy, 1993) 270 and calculating the reliability at threshold of a 95 percentile of the observation as follows:

$271 \quad$ Re liability $=\frac{1}{T} \sum_{b=1}^{B} n_{b}\left(\overline{p_{b}}-\overline{O_{b}}\right)^{2}$

272 To calculate the reliability, it is important to group the forecasts into $B$ forecast bins. Each bin

273 has a population of $n_{b}$, with an average forecast probability of $\overline{p_{b}}$ and an observed frequency of $274 \overline{O_{b}}$. For more details about reliability, readers are encouraged to refer to (Wilks, 2011). 


\section{3. Data and Study Area}

276 The applicability and usefulness of the methods are evaluated over three basins located in the

277 Western United States, as shown in Figure 2. The characteristics of each of these three basins are 278 summarized in Table 1.

279 Figure 2. The location of three study basins in the Western USA.

280 Table 1. Summary characteristics of the study basins.

281 This study is conducted on monthly precipitation over the historical period (1979-2014). The 282 first twenty-one years are used to calibrate the model, followed by fifteen years of validation 283 period. The observation dataset is extracted from the Parameter-Elevation Relationships on 284 Independent Slopes Model (PRISM) Climate Group, http://prism.oregonstate.edu. The observed 285 precipitation includes rainfall and melted snow over $4 \mathrm{~km}$ grid cells.

286 Climate Forecast System Reanalysis (CFSR) and Climate Forecast System version 2 (CFSv2) 287 developed by the National Centers for Environmental Prediction (NCEP) are utilized as the 288 climatologic forecast (Saha et al., 2010; Saha et al., 2014). The six-hourly precipitation at $0.5^{\circ}$ spatial 289 resolution is chosen and accumulated to the monthly values for evaluation of forecast.

290 To prepare the datasets for this study, the observation dataset is re-gridded to $0.5^{\circ} \times 0.5^{\circ}$ 291 resolution.

\section{4. Results and Discussion}

\section{4.1. Raw Forecast Validation}

294 We begin by evaluating the relationship between the raw forecast and the observation. In order to 295 achieve this objective, KGE is calculated for the raw forecast in relation to the observation for 296 calibration period. Results are shown in Figure 3. 
297 Figure 3. Kling-Gupta efficiency measure for the raw forecast during calibration period. White 298 grid cells in Rouge River Basin present missing observations.

299 The acceptable range for KGE is considered to be above 0.6; therefore, results in Figure 3 show 300 that the raw forecast does not have good skill as compared with the observation. More 301 specifically, results show that the KGE is less than 0.6 for the major portion of all three basins, 302 indicating that the observation mean is better in describing the precipitation event in comparison 303 to the raw forecast. The above inspections lead to the conclusion that it is not appropriate to use 304 the raw forecast.

\section{4.2. Selection of Copula Functions}

306 The experiment starts with finding the best copula function that describes the joint distribution 307 between the observation and the forecast during the calibration period. As an example, Figure 4 308 presents copula functions that successfully map the joint distribution for each month in each grid 309 cell in the upper Colorado River Basin. The figure demonstrates that among the copula functions 310 used in this basin, the Frank copula provides the best fit in describing the joint distribution

311 between the observation and the raw forecast in most months. Further inspection reveals that this 312 pattern is not followed for some of the months. As seen in Figure 4, the Clayton and T Copulas 313 are the most chosen distributions in March. In June, Gumbel and T-Copula are the dominant 314 copula functions chosen.

315 Figure 4. Selection of suitable copula functions for each grid cell across the Upper Colorado 316 River Basin.

317 According to Figure 4, copula functions will be fitted, and ensemble members will be built from 318 the conditional pdf of sample variable given the raw forecast at each time step. Here, the 319 ensemble precipitation forecast with twenty members will be generated. 


\subsection{Deterministic Verification}

321 The Absolute percent bias calculated for the study period (1979-2014) is presented in Figure 5.

322 This Figure demonstrates the calculated Absolute percent bias distribution over the study basins

323 for raw forecast and the bias-corrected ensemble means (i.e. MBG and COP-EPP). The highest

324 bias for the raw forecast is found in the Upper Colorado basin where Absolute percent bias 325 ranges from $50 \%$ to $300 \%$. Both techniques improve the accuracy of the raw forecast by more 326 than $50 \%$. The improvement is more considerable in Rogue basin, where the average of the 327 Absolute percent bias over the basin is reduced from $150 \%$ to $80 \%$ and $60 \%$ for MBG and COP328 EPP methods, respectively. COP-EPP method shows to be more effective in reducing the bias as 329 compared to MBG method. Overall, there is a $20 \%$ difference between the two techniques across 330 the study basins.

331 Figure 5. Absolute percent bias distribution over the three study basins. The bias is calculated 332 for the raw forecast (blue line), and the ensemble mean from the MGB and COP-EPP outputs 333 (red and green lines, respectively).

334 Results are evaluated with the KGE metric, which is a deterministic measure. A deterministic 335 comparison has been done on the ensemble means of both methods in calibration and validation 336 periods.

337 Figure 6 shows the KGE results in the calibration and the validation periods for each study basin. 338 Figures 3 and 6 show a significant improvement in generated forecast ensemble as compared to 339 the raw precipitation forecast. In addition, COP-EPP shows about $10 \%$ higher KGE values than 340 that of MBG during the calibration period. This is more significant for the validation period 341 where the range of calculated KGE for COP-EPP is always larger than that of MBG. 342 Furthermore, the discrepancy between the validation and the calibration period is less significant 
343 in the COP-EPP method than in the MBG method. For instance, in the Rogue River Basin, the 344 calculated KGE for the MBG method shows a decrease of $20 \%$ when compared with its 345 calibration results; while this is not the case for COP-EPP. In the Upper Colorado Basin, the 346 MBG method results in KGE values are less than 0.6 in some grids, which may not be 347 considered accurate enough in some specific studies (Bisselink, Zambrano-Bigiarini, Burek, \& 348 de Roo, 2016; Thiemig, Rojas, Zambrano-Bigiarini, \& De Roo, 2013).

349 Figure 6. KGE measure calculated over the study basins after post-processing using the COP350 EPP and MBG methods during calibration (left) and verification (right) periods.

\section{$351 \quad 4.4 . \quad$ Probabilistic Verification}

352 Figure 7 shows the results for the probabilistic verification of the ensemble forecast generated by 353 each method in each basin. This figure presents the CRPSS analysis for both the calibration and 354 the validation periods. The results of the CRPSS analysis demonstrate a significant improvement 355 of the raw forecast after EPP in both techniques, with a more significant improvement based on 356 COP-EPP. During the calibration period, the copula method shows superior results by almost $35710 \%$ in comparison to the MBG's results in all basins, whereas in Rogue River Basin, disparity 358 in the MBG results is slightly higher, indicating that in basins with higher precipitation, COP359 EPP is more successful than MBG in generating more skillful ensemble forecasts.

360 Figure 7 indicates that both methods result in higher values of CRPSS in the western parts of 361 Upper Colorado River Basin. For central parts of Southern Snake Basin, CRPSS shows the 362 lowest values for the generated ensemble precipitation compared to the observation. 363 Significantly, the spatial pattern is maintained in the validation period for both techniques in all 364 the basins. 
365 Comparing the results for both techniques in the validation period indicates that copula outputs

366 are more successful in preserving the accuracy of the generated ensemble in this period. For

367 instance, in the case of Rogue River Basin, CRPSS is reduced by almost $2 \%$ in the validation

368 period compared to the calibration for COP-EPP outputs. Meanwhile, this difference is about

$36910 \%$ for MBG results. This schema is visible for the other two basins with less noticeable

370 difference.

371 Figure 7. CRPSS measure calculated for 3 basins after implementing two post-processing

372 methods for the calibration (left) and verification (right) periods.

373 The ROC scores are presented in Figure 8. Both post-processing methods maintain a ROC score

374 above 0.5 , which is an encouraging outcome for the generated ensemble forecast. Comparing the

375 results from the Southern Snake and Upper Colorado basins shows that COP-EPP generates the 376 ensemble forecast with higher resolution than that of the MBG method. The difference between

377 the two methods is more noticeable in Rogue River Basin, which receives relatively higher 378 precipitation than the other basins.

379 Figure 8. Assessment of forecast resolution based on ROC score for winter (Dec, Jan, and Feb) 380 precipitation during verification period (2001-2014).

381 Since the focus of this study is to minimize the bias in the forecast, in addition to resolution, 382 reliability of the generated forecast is evaluated for each basin. According to the formulation of 383 reliability (eq. 21), the lower the score the more reliable the ensemble forecast will be. Figure 9 384 illustrates that COP-EPP has a reliability score of less than 0.05 for approximately all the grid 385 cells in the three basins. Although, in general, the MBG method, provides a reliable ensemble 386 forecast across the basins, the COP-EPP shows noticeably better performance than the MBG . 387 Since the reliability of the forecasts is examined for the ninety-fifth percentile of the observation, 
388 this can also indicate the reliability of the generated forecasts on the extremes. Although both 389 methods are shown capable of detecting the extremes, the COP-EPP demonstrates more accuracy 390 on extreme values.

391 Figure 9. Reliability measure for winter precipitation (Dec, Jan, and Feb) calculated at $95^{\text {th }}$ 392 percentile of observation during the verification period (2001-2014). This measure ranges from 0 393 to 1 with the optimal value of 0.

\section{$394 \quad$ 4.5. Seasonal Evaluation of the Generated Ensemble Forecasts}

395 To study the performance of the generated ensemble precipitation based on the COP-EPP, 396 seasonal precipitation is evaluated for each basin. Accordingly, the most probable ensemble 397 member (the mode of the conditional pdf), which can substitute the raw forecast as a better 398 predictor, is chosen for this purpose. For a clear inspection, the spatial average of the most 399 probable ensemble member is utilized over the study basin.

400 Results of seasonal assessment are summarized in Figure 10. In the figure, the observation is 401 shown by the green line, the raw forecast is shown by the red line, the blue line displays the most 402 probable ensemble member from the generated ensemble forecast by COP-EPP, and the black 403 line represents the indicated ensemble member from MBG's generated forecast. Overall, the 404 post-processed forecast shows a significant improvement in describing seasonal precipitation 405 distribution in comparison to the raw forecast. For an instance, in the spring, the precipitation 406 distribution from the raw forecast (red line) overestimates the observation (green line), whereas 407 the COP-EPP forecast almost follows the same distribution as the observation. In summer, the 408 raw forecast distribution shows better performance compared to other seasons. On the other 409 hand, in a severe condition such as Rogue River Basin, which is a coastal region, the raw 
410 forecast is not able to follow the observation's distribution. This is more noticeable in winter 411 when basins receive more precipitation.

412 Seasonal results for the Upper Colorado Basin, a semi-arid region, demonstrate no significant 413 difference between precipitations in different seasons whereas in the Southern Snake Basin, 414 spring and winter receive more precipitation than summer and fall. These changes become more 415 significant when a coastal basin, such as the Rogue River Basin, is studied. In inspecting the 416 results for the Upper Colorado Basin, it can be seen that the raw forecast is over-predicting the 417 observation, especially in spring and winter. This pattern is approximately the same for the 418 Southern Snake River Basin. However, in the Rogue River Basin, summers receive much less 419 precipitation compared to other seasons. Observations for this basin show the same distribution 420 pattern for a high level of precipitation in fall and spring. Results for winter in this basin show 421 much higher precipitation with an average of about $600 \mathrm{~mm}$. In comparing the raw forecast with 422 the three other datasets, i.e. observation and two generated forecasts, results indicate that the raw 423 forecast has higher seasonal precipitation values in all seasons with a significant difference in 424 winter. In summer, the forecast is following the same distribution as the observation with minor 425 under-prediction for the Rogue River Basin. Furthermore, in wet seasons, the generated forecasts 426 successfully follow the observation distribution, whereas MBG is showing minor under427 prediction. In extreme cases, such as winter in the Rogue River Basin, the raw forecast shows a 428 large bias while COP-EPP was successful in reducing the bias, similar to spring and fall.

429 Overall, COP-EPP has shown significant potential in generating more reliable and accurate 430 ensemble forecasts. Compared to the benchmark technique, COP-EPP shows more consistency 431 in the validation period as compared to that of MBG. In the cases with extreme precipitation, 432 COP-EPP still shows superior results. Lastly, COP-EPP is robust in conserving the spatial 
433 pattern of calculated measures. Generally, results show higher accuracy in wet seasons; a 434 specifically generated ensemble forecast is showing promising results in the extreme cases such 435 as winter for the Rogue River Basin.

436 Brown and Seo (2013) argue that back and forth transformation from Gaussian space can 437 invalidate the optimality of estimated parameters of the conditional probability distribution. 438 However, the improvement in the COP-EPP compared to the MBG can be attributed to the 439 procedure used by COP-EPP to model the dependence structure between observation and 440 forecast. COP-EPP joins the variables via their marginal distribution. Therefore, the complexity 441 in the hydro-meteorological processes does not constrain modeling the joint behavior of 442 observation and forecast (Dupuis, 2007; Madadgar \& Moradkhani, 2014).

443 Figure 10. Probability density functions of seasonal precipitation from the observation, raw 444 forecast, and most probable forecast based on the COP-EPP and MBG approach for three study 445 basins. Seasonal precipitation is spatially averaged over all grid cells of each basin. Seasons are 446 categorized in the following order: Spring (Mar, Apr, and May), summer (Jun, Jul, and Aug), fall 447 (Sep, Oct, and Nov), and winter (Dec, Jan, and Feb).

\section{5. Conclusion and Summary}

449 The purpose of this study is to examine the accuracy and reliability of ensemble precipitation 450 prediction utilizing copula functions. The technique is based on the relationship between the 451 single value forecast and historical observation. Therefore, the assessment is done by comparing 452 results from the new copula method with that of a well-known procedure, MBG.

453 Comparison is undertaken by employing three different basins with semi-arid to coastal climates 454 to study the performance of the techniques in different climate regimes. Deterministic 
455 verification indicates promising improvement in the mean ensemble using the COP-EPP for 456 generating ensemble precipitation forecast. In order to assess the forecast skill, probabilistic 457 measures including CRPSS, reliability, and the ROC score are employed. The results of CRPSS 458 indicate that the generated ensemble forecast from COP-EPP is more reliable and accurate in 459 comparison to the meta-Gaussian one. Furthermore, through analysis of reliability, it is noticed 460 that the copula- based method is more successful in generating the ensemble forecasts that 461 represent extremes. The ROC score indicated that both techniques are capable of generating 462 potentially useful ensemble forecasts with high resolution; however, in the basin with higher 463 precipitation (i.e., Rogue River Basin), COP-EPP proves to be even more superior.

464 Overall, both techniques show promising results, and existing procedures generated ensembles 465 with acceptable reliability. However, using copula functions will help improve the quality of 466 ensemble forecasting. COP-EPP is shown to be more precise in building the ensemble 467 precipitation forecast. In other words, results demonstrate that the copula procedure is 468 approximately independent of spatial and temporal changes in the data.

469 It is worth mentioning that incorporating copula functions into EPP helps overcome the 470 assumption of normal distribution for the observation and forecast. It is therefore possible to 471 eliminate the transformation step in EPP procedure. Moreover, copula functions are capable of 472 building joint distribution between two datasets with any level of dependency, and for any 473 marginal distributions. These characteristics of copula functions help us generate more accurate 474 ensemble forecast.

\section{Acknowledgement}

476 The authors would like to acknowledge the financial support for this study by NOAA Grants No. $477 \quad$ NA11NWS4680002 and NA140AR4310234. 


\section{References}

Aho, K., Derryberry, D. and Peterson, T., 2014. Model selection for ecologists: the worldviews of AIC and BIC. Ecology, 95(3): 631-636.

Anderson, T.W., 1962. On the distribution of the two-sample Cramer-von Mises criterion. The Annals of Mathematical Statistics: 1148-1159.

Bisselink, B., Zambrano-Bigiarini, M., Burek, P., de Roo, A., 2016. Assessing the role of uncertain precipitation estimates on the robustness of hydrological model parameters under highly variable climate conditions. J. Hydrol. Reg. Stud. 8, 112-129. doi:http://dx.doi.org/10.1016/j.ejrh.2016.09.003

Bourgin, F., Ramos, M.H., Thirel, G. and Andréassian, V., 2014. Investigating the interactions between data assimilation and post-processing in hydrological ensemble forecasting. Journal of Hydrology, 519, Part D: 2775-2784.

Brown, J.D. and Seo, D.J., 2013. Evaluation of a nonparametric post-processor for bias correction and uncertainty estimation of hydrologic predictions. Hydrological Processes, 27(1): 83-105.

Bárdossy, A., 2006. Copula-based geostatistical models for groundwater quality parameters. Water Resources Research, 42(11).

Bárdossy, A. and Pegram, G., 2013. Interpolation of precipitation under topographic influence at different time scales. Water Resources Research, 49(8): 4545-4565.

Bárdossy, A. and Pegram, G., 2014. Infilling missing precipitation records - A comparison of a new copula-based method with other techniques. Journal of Hydrology, 519, Part A: 1162-1170.

Clark, M., Gangopadhyay, S., Hay, L., Rajagopalan, B. and Wilby, R., 2004. The Schaake shuffle: A method for reconstructing space-time variability in forecasted precipitation and temperature fields. Journal of Hydrometeorology, 5(1): 243-262.

Clark, M.P. and Hay, L.E., 2004. Use of medium-range numerical weather prediction model output to produce forecasts of streamflow. Journal of Hydrometeorology, 5(1): 15-32.

Clark, M.P. and Vrugt, J.A., 2006. Unraveling uncertainties in hydrologic model calibration: Addressing the problem of compensatory parameters. Geophysical Research Letters, 33(6).

DeChant, C.M. and Moradkhani, H., 2014b. Toward a reliable prediction of seasonal forecast uncertainty: Addressing model and initial condition uncertainty with ensemble data assimilatio $\mathrm{n}$ and Sequential Bayesian Combination. Journal of Hydrology, 519, Part D(0): 2967-2977.

DeChant, C.M. and Moradkhani, H., 2015. On the assessment of reliability in probabilistic hydrometeorological event forecasting. Water Resources Research.

Duan, Q., Ajami, N.K., Gao, X. and Sorooshian, S., 2007. Multi-model ensemble hydrologic prediction using Bayesian model averaging. Advances in Water Resources, 30(5): 13711386.

Dung, N.V., Merz, B., Bárdossy, A. and Apel, H., 2015. Handling uncertainty in bivariate quantile estimation - An application to flood hazard analysis in the Mekong Delta. Journal of Hydrology, 527: 704-717.

Dupuis, D. J. (2007). Using copulas in hydrology: Benefits, cautions, and issues. Journal of Hydrologic Engineering, 12(4), 381-393.

Favre, A.C., El Adlouni, S., Perreault, L., Thiémonge, N. and Bobée, B., 2004. Multivariate 
hydrological frequency analysis using copulas. Water resources research, 40(1).

Genest, C., Rémillard, B. and Beaudoin, D., 2009. Goodness-of-fit tests for copulas: A review and a power study. Insurance: Mathematics and economics, 44(2): 199-213.

Gupta, H.V., Kling, H., Yilmaz, K.K. and Martinez, G.F., 2009. Decomposition of the mean squared error and NSE performance criteria: Implications for improving hydrological modelling. Journal of Hydrology, 377(1-2): 80-91.

Gupta, H.V., Sorooshian, S., Yapo, P.O., 1999. Status of automatic calibration for hydrologic models: Comparison with multilevel expert calibration. J. Hydrol. Eng. 4, 135-143.

Hamill, T.M. and Whitaker, J.S., 2006. Probabilistic quantitative precipitation forecasts based on reforecast analogs: Theory and application. Monthly Weather Review, 134(11): 32093229.

Hamill, T.M., Whitaker, J.S. and Mullen, S.L., 2006. Reforecasts: An Important Dataset for Improving Weather Predictions. Bulletin of the American Meteorological Society, 87(1): 33-46.

Hersbach, H., 2000. Decomposition of the Continuous Ranked Probability Score for Ensemble Prediction Systems. Weather and Forecasting, 15(5): 559-570.

Joe, H., 1997. Multivariate models and multivariate dependence concepts, 73. CRC Press.

Kavetski, D., Kuczera, G. and Franks, S.W., 2006. Bayesian analysis of input uncertainty in hydrological modeling: 2. Application. Water Resources Research, 42(3).

Kelly, K.S. and Krzysztofowicz, R., 1997. A bivariate meta-Gaussian density for use in hydrology. Stochastic Hydrology and Hydraulics, 11(1): 17-31.

Krzysztofowicz, R. and Herr, H.D., 2001. Hydrologic uncertainty processor for probabilistic river stage forecasting: precipitation-dependent model. Journal of Hydrology, 249(1): 4668.

Kumar, A., Chen, M., Zhang, L., Wang, W., Xue, Y., Wen, C., Marx, L. and Huang, B., 2012. An analysis of the nonstationarity in the bias of sea surface temperature forecasts for the NCEP Climate Forecast System (CFS) version 2. Monthly Weather Review, 140(9): 3003-3016.

Li, H., Luo, L., Wood, E.F. and Schaake, J., 2009. The role of initial conditions and forcing uncertainties in seasonal hydrologic forecasting. Journal of Geophysical Research: Atmospheres (1984-2012), 114(D4).

Madadgar, S. and Moradkhani, H., 2013. A Bayesian Framework for Probabilistic Seasonal Drought Forecasting. Journal of Hydrometeorology, 14(6): 1685-1705.

Madadgar, S. and Moradkhani, H., 2014a. Improved Bayesian multimodeling: Integration of copulas and Bayesian model averaging. Water Resources Research: n/a-n/a.

Madadgar, S. and Moradkhani, H., 2014b. Spatio-temporal drought forecasting within Bayesian networks. Journal of Hydrology, 512: 134-146.

Madadgar, S., Moradkhani, H. and Garen, D., 2014. Towards improved post-processing of hydrologic forecast ensembles. Hydrological Processes, 28(1): 104-122.

Mason, I., 1982. A model for assessment of weather forecasts. Aust. Meteor. Mag, 30(4): 291303.

Murphy, A.H., 1993. What is a good forecast? An essay on the nature of goodness in weather forecasting. Weather and forecasting, 8(2): 281-293.

Moriasi, D.N., Arnold, J.G., Van Liew, M.W., Bingner, R.L., Harmel, R.D., Veith, T.L., 2007. Model evaluation guidelines for systematic quantification of accuracy in watershed simulations. Trans. Asabe 50, 885-900. 
Najafi, M.R. and Moradkhani, H., 2015. Ensemble combination of seasonal streamflow forecasts. Journal of Hydrologic Engineering, 21(1): 04015043.

Nelsen, R.B., 1999. An introduction to copulas, volume 139 of Lecture Notes in Statistics. Springer-Verlag, New York.

Park, Y.Y., Buizza, R. and Leutbecher, M., 2008. TIGGE: Preliminary results on comparing and combining ensembles. Quarterly Journal of the Royal Meteorological Society, 134(637): 2029-2050.

Parrish, M.A., Moradkhani, H. and DeChant, C.M., 2012. Toward reduction of model uncertainty: Integration of Bayesian model averaging and data assimilation. Water Resources Research, 48(3): n/a-n/a.

Peng, P., Barnston, A.G. and Kumar, A., 2013. A comparison of skill between two versions of the NCEP climate forecast system (CFS) and CPC's operational short-lead seasonal outlooks. Weather and Forecasting, 28(2): 445-462.

Raftery, A.E., 1986. Choosing models for cross-classifications. American Sociological Review, 51(1): 145-146.

Raleigh, M.S., Lundquist, J.D. and Clark, M.P., 2015. Exploring the impact of forcing error characteristics on physically based snow simulations within a global sensitivity analysis framework. Hydrol. Earth Syst. Sci., 19(7): 3153-3179.

Rayner, S., Lach, D. and Ingram, H., 2005. Weather Forecasts are for Wimps: Why Water Resource Managers Do Not Use Climate Forecasts. Climatic Change, 69(2-3): 197-227.

Robertson, D.E., Shrestha, D.L. and Wang, Q.J., 2013. Post processing rainfall forecasts from numerical weather prediction models for short term streamflow forecasting. Hydrology and Earth System Sciences Discussions, 10(5): 6765-6806.

Roulin, E., \& Vannitsem, S. (2012). Postprocessing of ensemble precipitation predictions with extended logistic regression based on hindcasts. Monthly Weather Review, 140(3), 874-888.

Saha, S., Moorthi, S., Pan, H.-L., Wu, X., Wang, J., Nadiga, S., Tripp, P., Kistler, R., Woollen, J. and Behringer, D., 2010. The NCEP climate forecast system reanalysis. Bulletin of the American Meteorological Society, 91(8): 1015-1057.

Saha, S., Moorthi, S., Wu, X., Wang, J., Nadiga, S., Tripp, P., Behringer, D., Hou, Y.-T., Chuang, H.-y. and Iredell, M., 2014. The NCEP climate forecast system version 2. Journal of Climate, 27(6): 2185-2208.

Salvadori, G. and De Michele, C., 2010. Multivariate multiparameter extreme value models and return periods: A copula approach. Water resources research, 46(10).

Schaake, J., Demargne, J., Hartman, R., Mullusky, M., Welles, E., Wu, L., Herr, H., Fan, X. and Seo, D.J., 2007. Precipitation and temperature ensemble forecasts from single-value forecasts. Hydrology and Earth System Sciences Discussions Discussions, 4(2): 655-717.

Sklar, M., 1959. Fonctions de répartition à n dimensions et leurs marges. Université Paris 8.

Steinschneider, S., Polebitski, A., Brown, C. and Letcher, B.H., 2012. Toward a statistical framework to quantify the uncertainties of hydrologic response under climate change. Water Resources Research, 48(11).

Stephens, M.A., 1974. EDF statistics for goodness of fit and some comparisons. Journal of the American statistical Association, 69(347): 730-737.

Sumner, G., Homar, V., \& Ramis, C. (2001). Precipitation seasonality in eastern and southern coastal Spain. International Journal of Climatology, 21(2), 219-247. http://doi.org/10.1002/joc.600.

Tao, Y., Duan, Q., Ye, A., Gong, W., Di, Z., Xiao, M. and Hsu, K., 2014. An evaluation of post- 
processed TIGGE multimodel ensemble precipitation forecast in the Huai river basin. Journal of Hydrology, 519, Part D(0): 2890-2905.

Thiemig, V., Rojas, R., Zambrano-Bigiarini, M., \& De Roo, A. (2013). Hydrological evaluation of satellite-based rainfall estimates over the Volta and Baro-Akobo Basin. Journal of Hydrology, 499, 324-338.

Wang, Q.J. and Robertson, D.E., 2011. Multisite probabilistic forecasting of seasonal flows for streams with zero value occurrences. Water Resources Research, 47(2): W02546.

Wang, Q.J., Robertson, D.E. and Chiew, F.H.S., 2009. A Bayesian joint probability modeling approach for seasonal forecasting of streamflows at multiple sites. Water Resources Research, 45(5): W05407.

Wang, W., Hung, M.-P., Weaver, S.J., Kumar, A. and Fu, X., 2014. MJO prediction in the NCEP Climate Forecast System version 2. Climate dynamics, 42(9-10): 2509-2520.

Wilks, D. S. 2009. Extending logistic regression to provide full-probability-distribution MOS forecasts. Meteorological Applications, 16(3), 361-368.

Wilks, D.S., 2011. Statistical methods in the atmospheric sciences, 100. Academic press.

Wood, A.W. and Schaake, J.C., 2008. Correcting errors in streamflow forecast ensemble mean and spread. Journal of Hydrometeorology, 9(1): 132-148.

Wu, L., Seo, D.-J., Demargne, J., Brown, J.D., Cong, S. and Schaake, J., 2011. Generation of ensemble precipitation forecast from single-valued quantitative precipitation forecast for hydrologic ensemble prediction. Journal of hydrology, 399(3): 281-298.

Yan, H., DeChant, C.M. and Moradkhani, H., Improving Hydrologic Data Assimilation by a Multivariate Particle Filter-Markov Chain Monte Carlo, pp. 0773.

Ye, A., Duan, Q., Yuan, X., Wood, E.F. and Schaake, J., 2014. Hydrologic post-processing of MOPEX streamflow simulations. Journal of Hydrology, 508: 147-156.

Zehe, E. and Blöschl, G., 2004. Predictability of hydrologic response at the plot and catchment scales: Role of initial conditions. Water Resources Research, 40(10).

Zhao, L., Duan, Q., Schaake, J., Ye, A. and Xia, J., 2011. A hydrologic post-processor for ensemble streamflow predictions. Advances in Geosciences, 29(29): 51-59.

Zhao, T., Wang, Q.J., Bennett, J.C., Robertson, D.E., Shao, Q. and Zhao, J., 2015. Quantifying predictive uncertainty of streamflow forecasts based on a Bayesian joint probability model. Journal of Hydrology, 528: 329-340. 

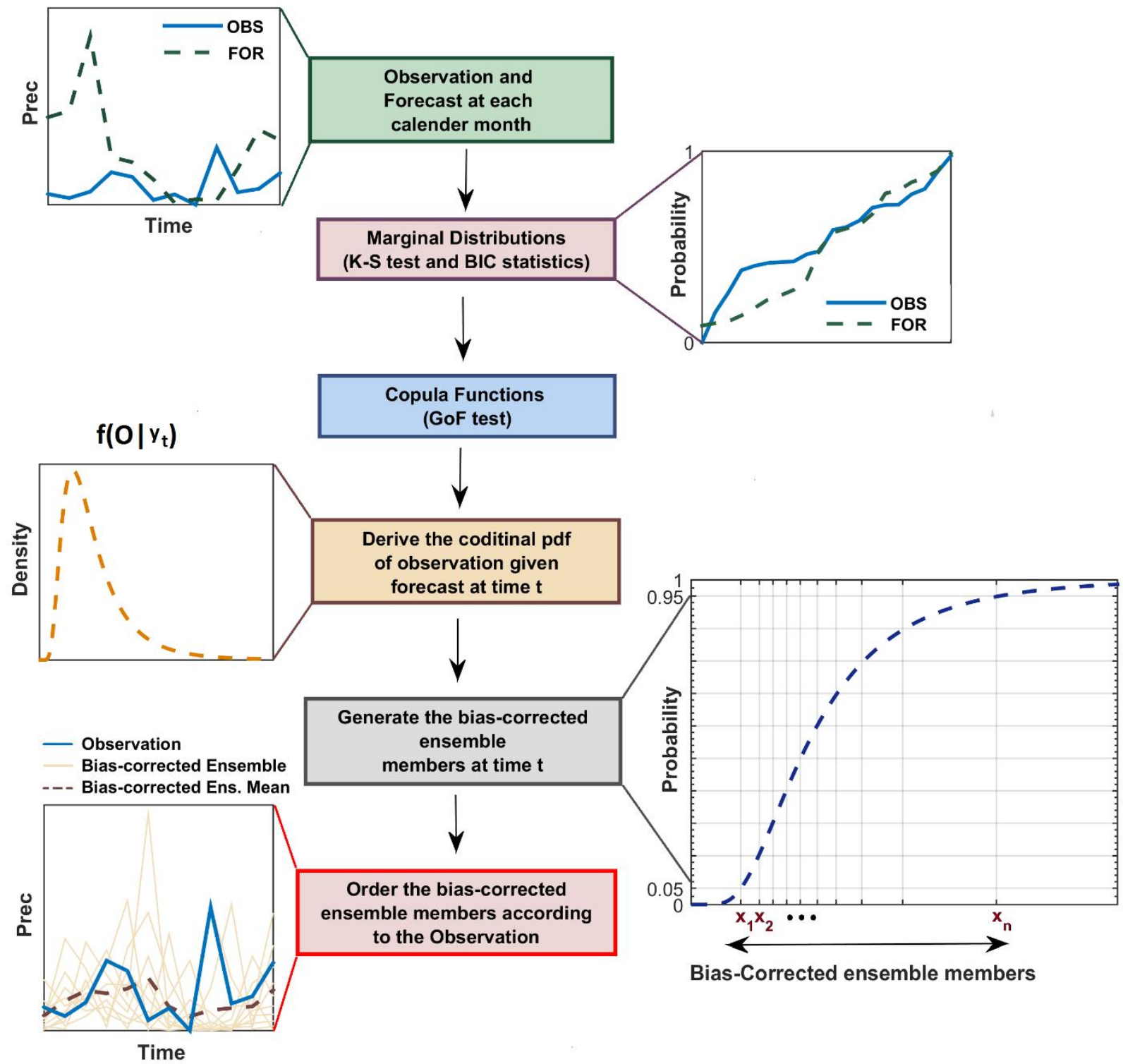

Figure 1. Schematic of the Copula-based ensemble post-processing (COP-EPP). The ensemble members are generated by sampling from conditional pdf and reconstructed according to Schaake shuffle. 


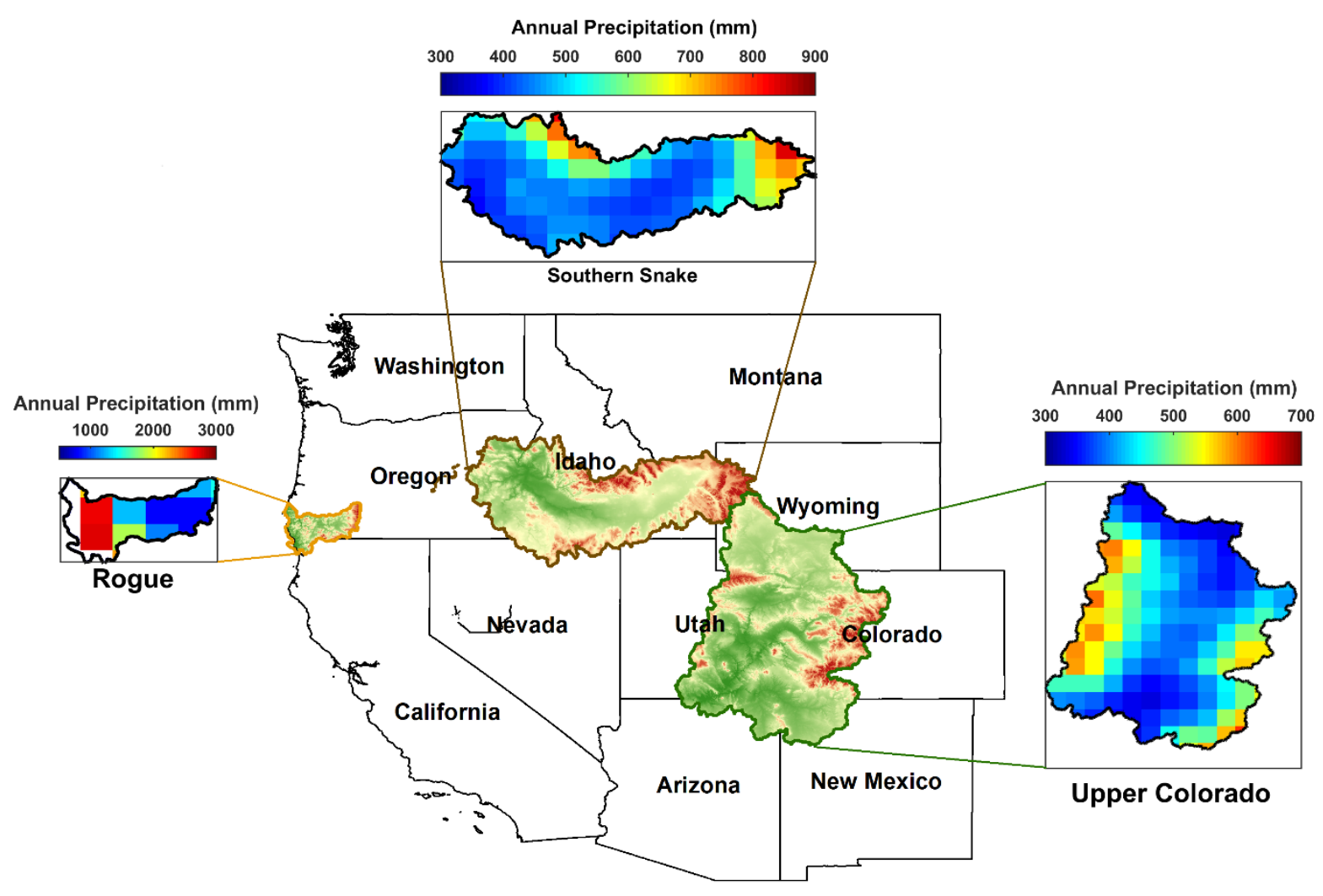

Figure 2. The location of three study basins in the Western USA. 

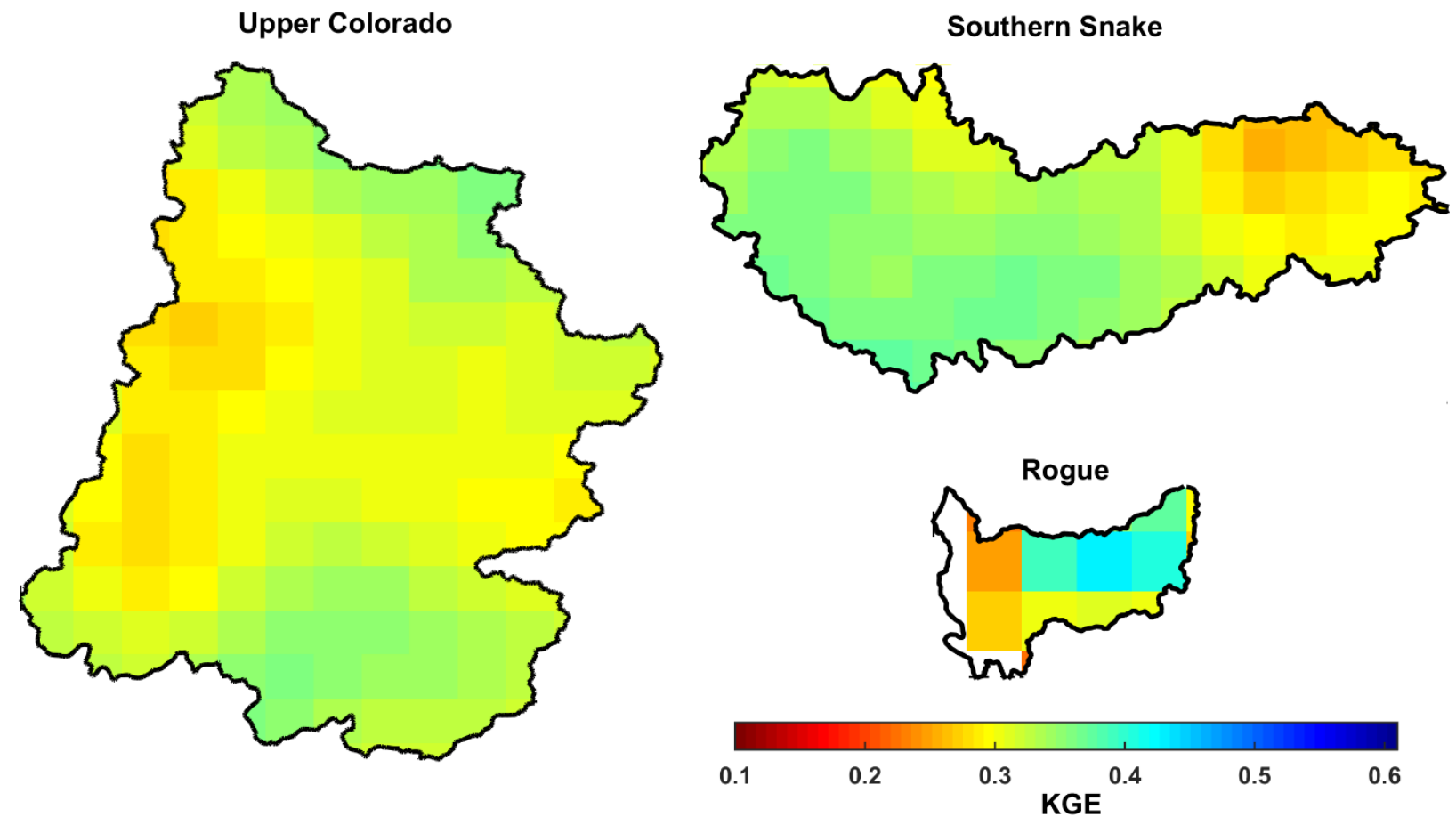

Figure 3. Kling-Gupta efficiency measure for the raw forecast during calibration period. White grid cells in Rouge River Basin present missing observations. 

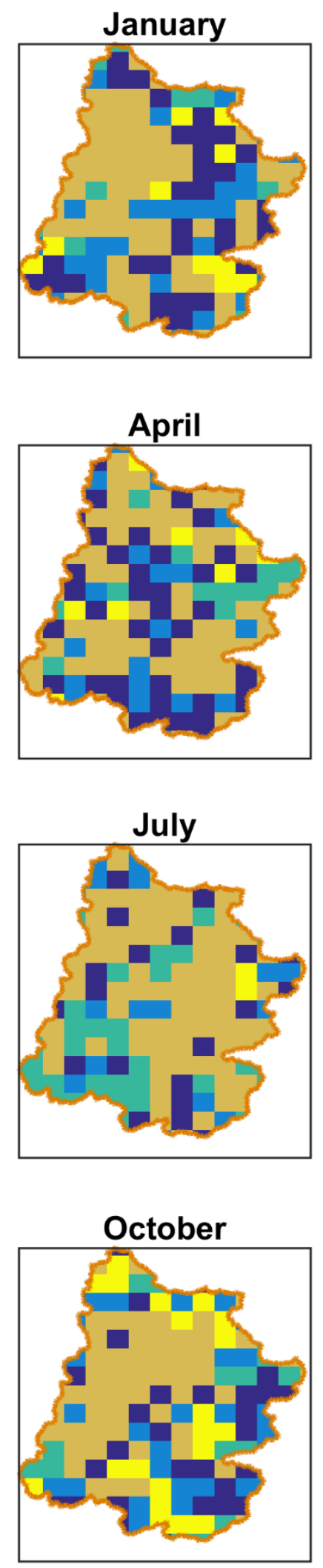

Tcopula
February

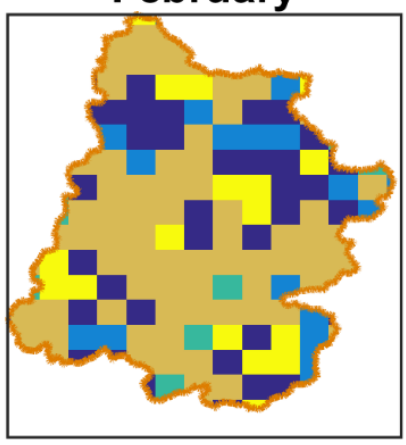

May
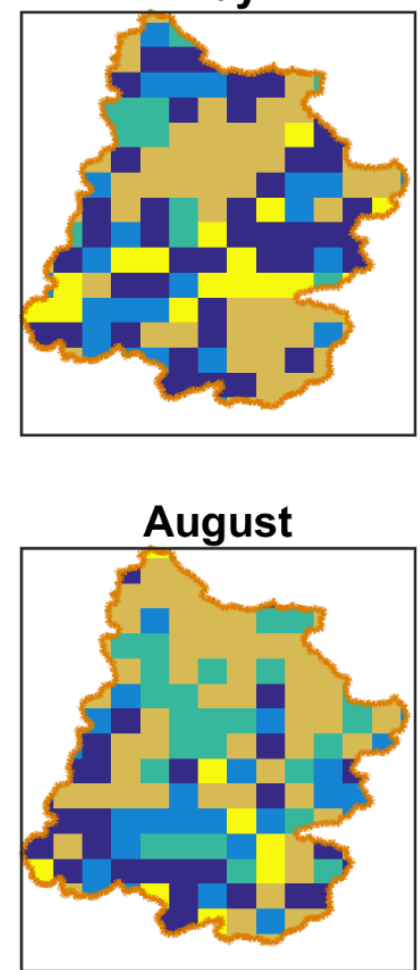

November

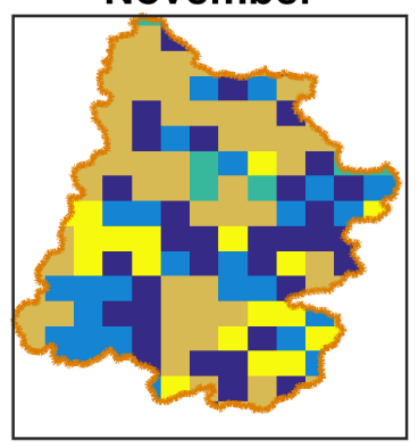

March
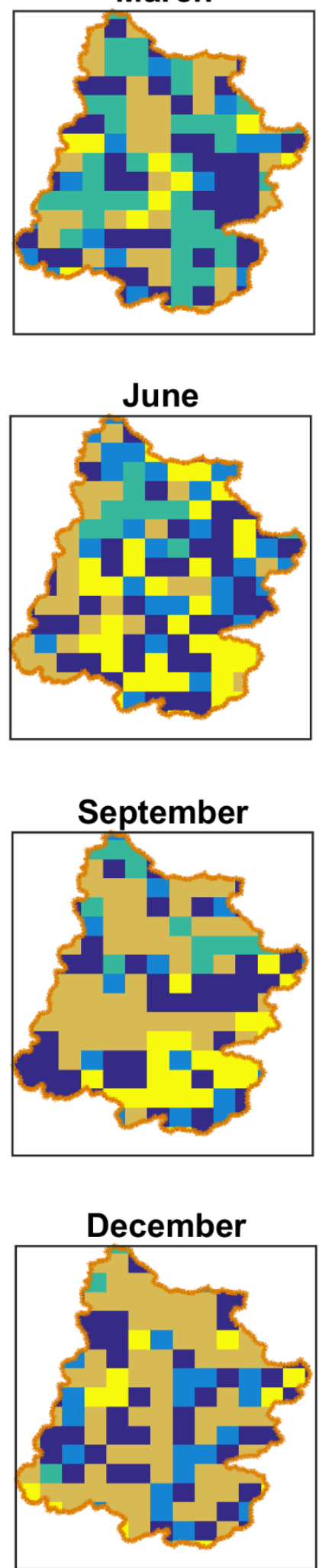

\begin{tabular}{|c|c|c|c|}
\hline Tcopula & Normal & Clayton & Frank \\
\hline
\end{tabular}


Figure 4. Selection of suitable copula functions for each grid cell across the Upper Colorado River Basin.
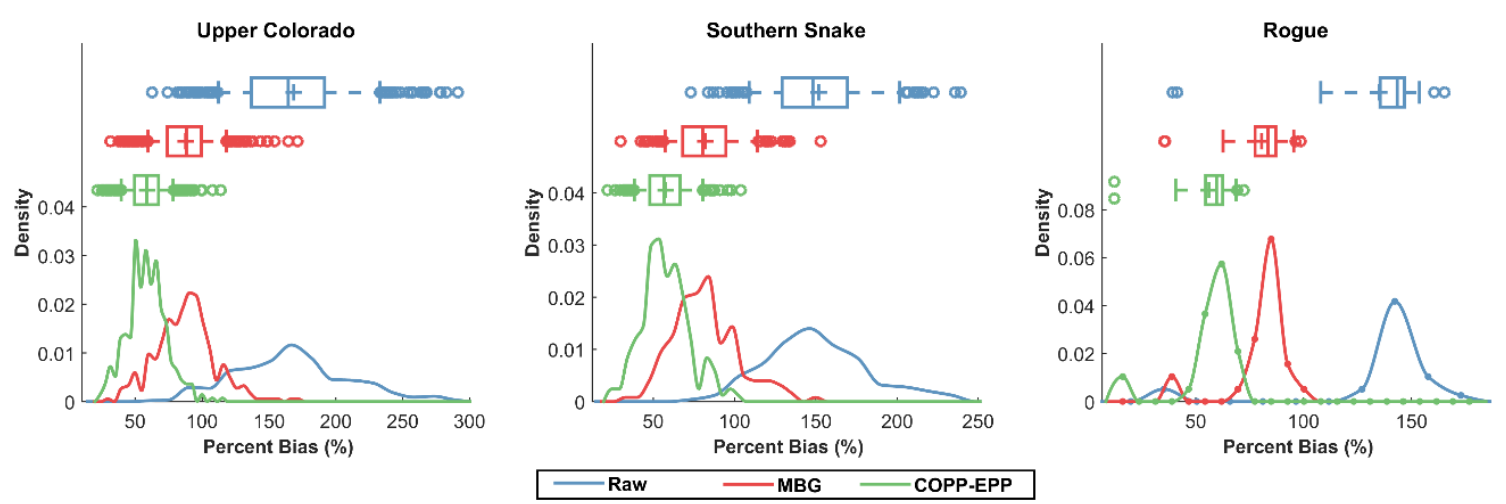

Figure 5. Absolute percent bias distribution over the three study basins. The bias is calculated for the raw forecast (blue line), and the ensemble mean from the MGB and COP-EPP outputs (red and green lines, respectively). 

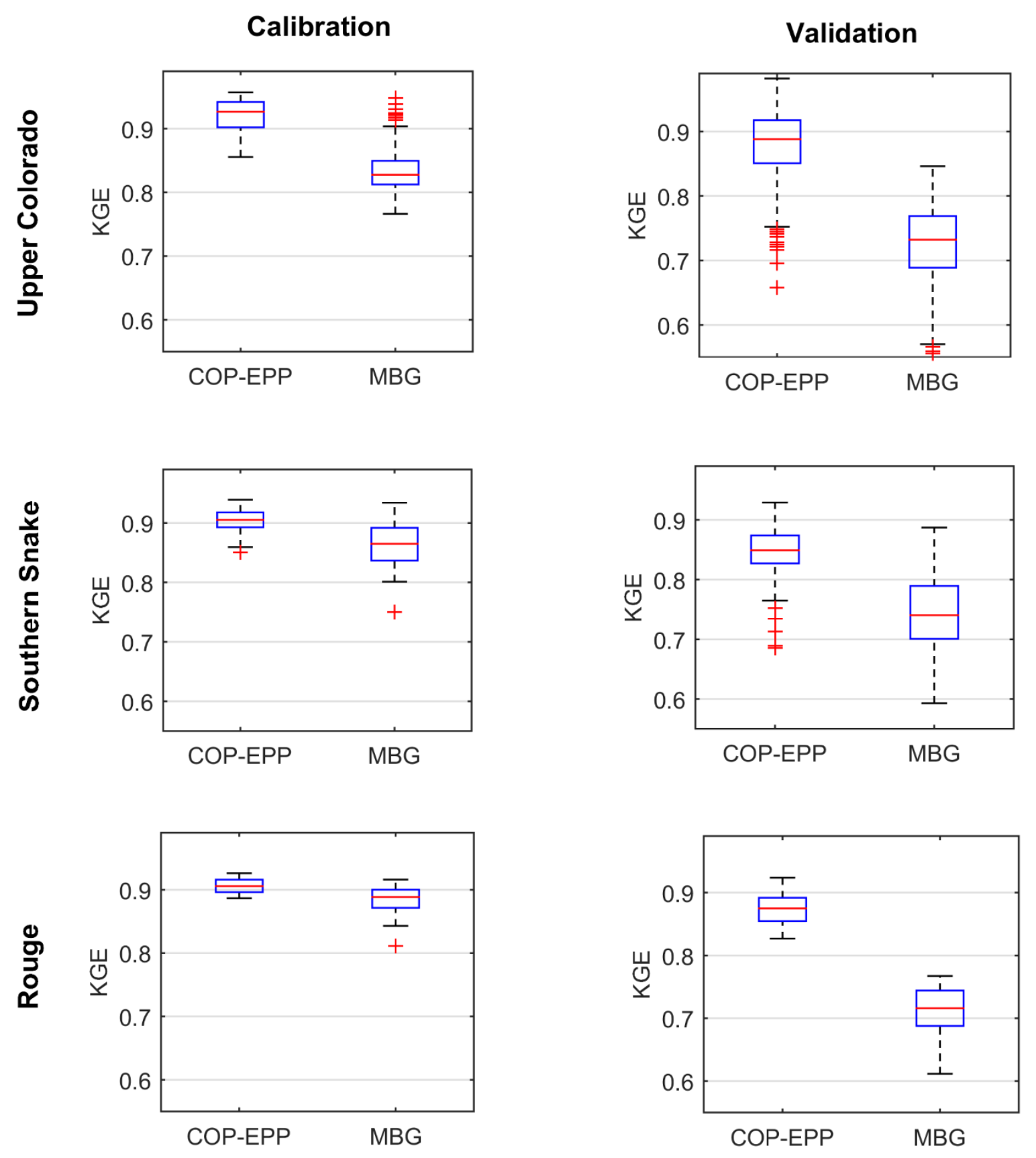

Figure 6. KGE measure calculated over the study basins after post-processing using the COPEPP and MBG methods during calibration (left) and verification (right) periods. 

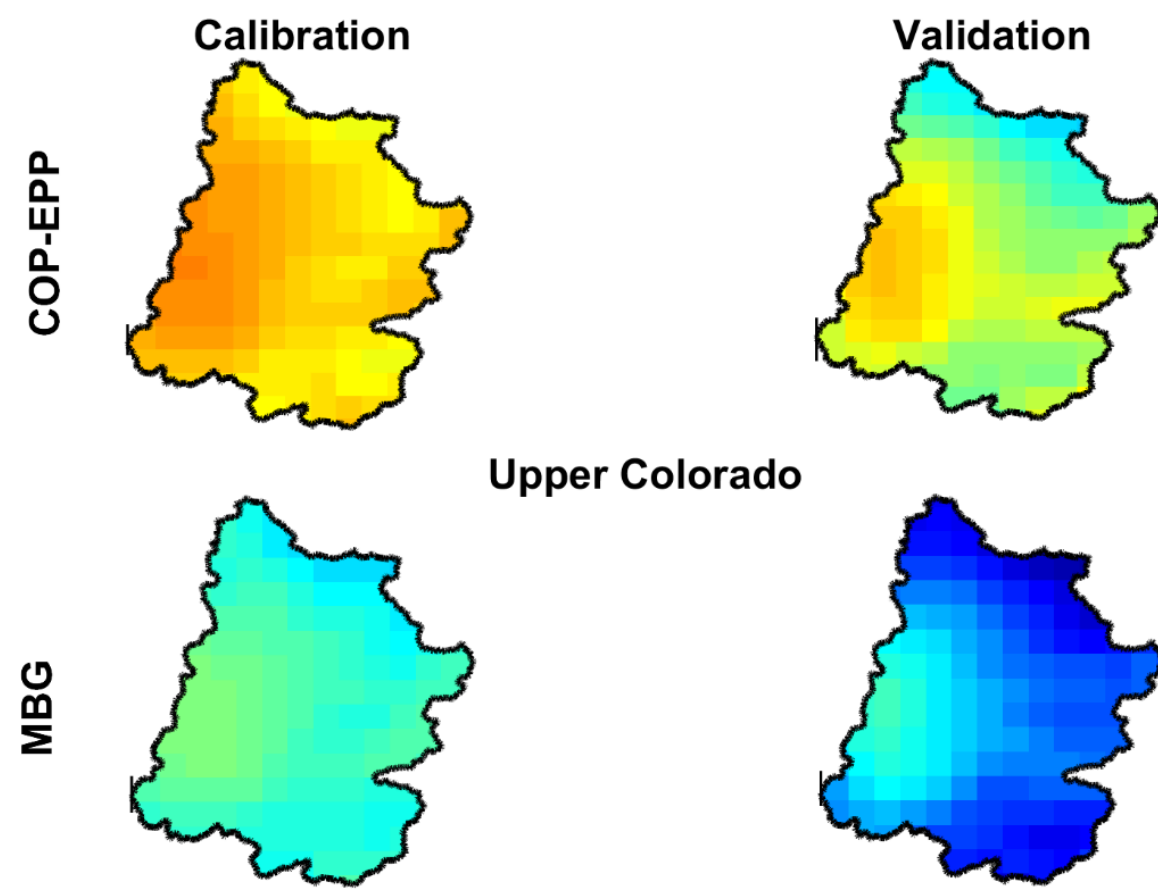

\section{Upper Colorado}
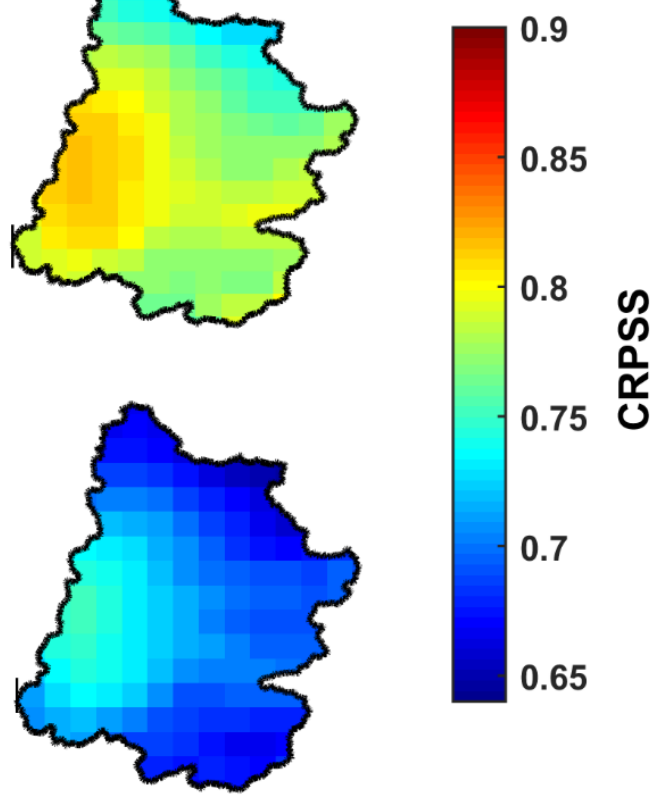

0.7

0.65
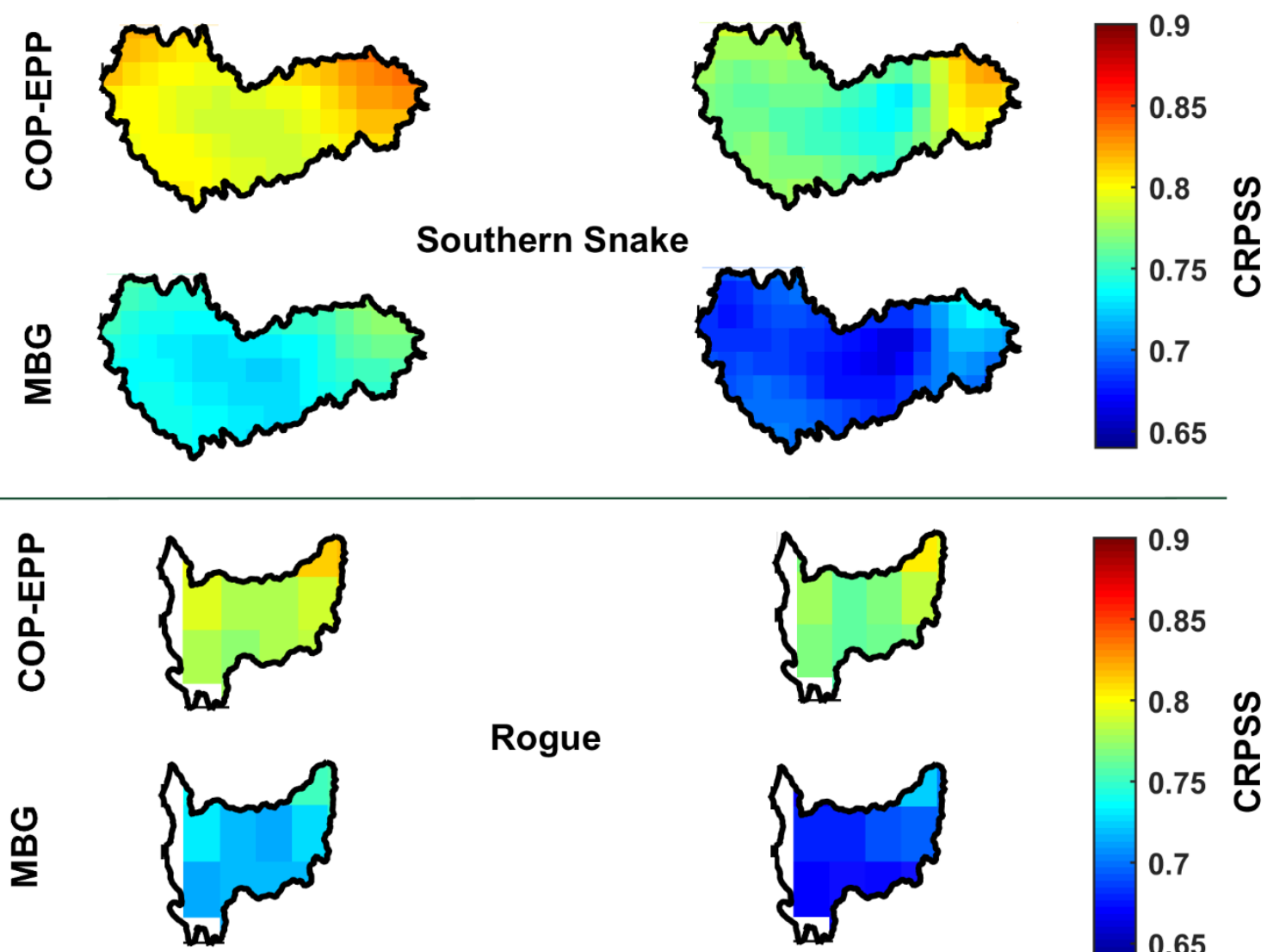

0.9

0.85

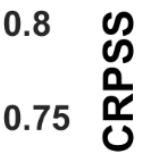

0.7

0.65 
Figure 7. CRPSS measure calculated for 3 basins after implementing two post-processing methods for the calibration (left) and verification (right) periods.
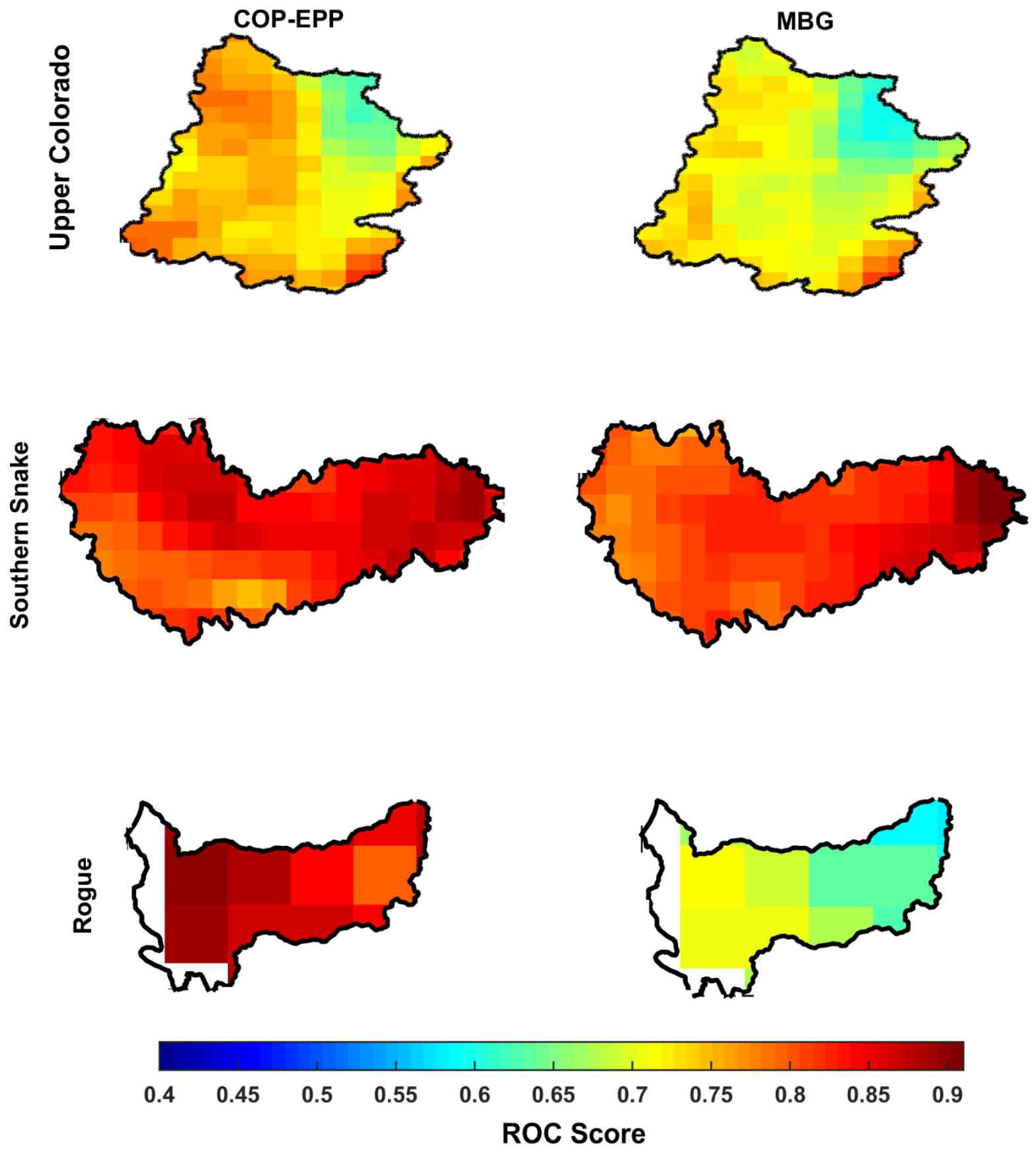
Figure 8. Assessment of forecast resolution based on ROC score for winter (Dec, Jan, and Feb) precipitation during verification period (2001-2014). 

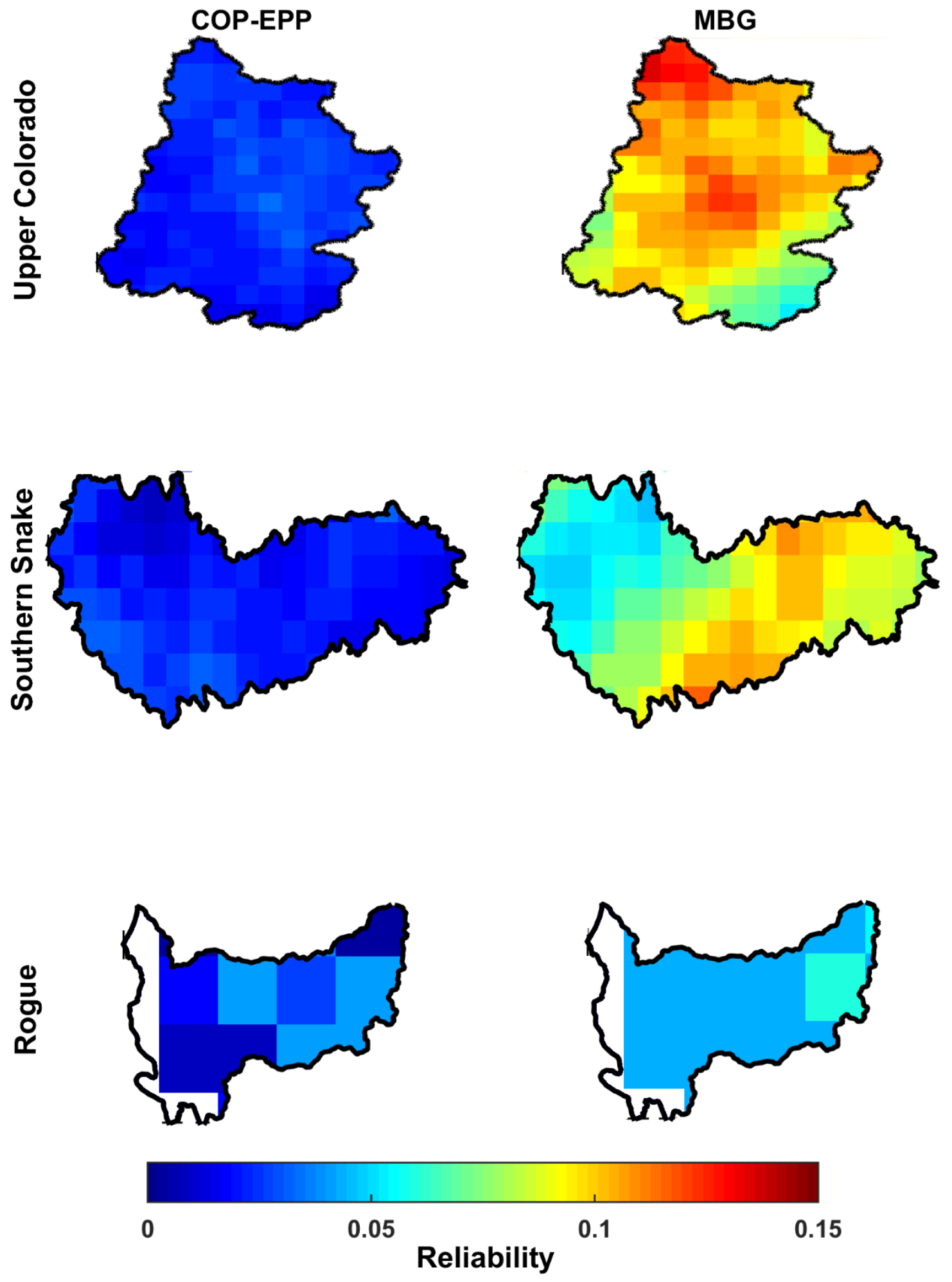
Figure 9. Reliability measure for winter precipitation (Dec, Jan, and Feb) calculated at $95^{\text {th }}$ percentile of observation during the verification period (2001-2014). This measure ranges from 0 to 1 with the optimal value of 0 .
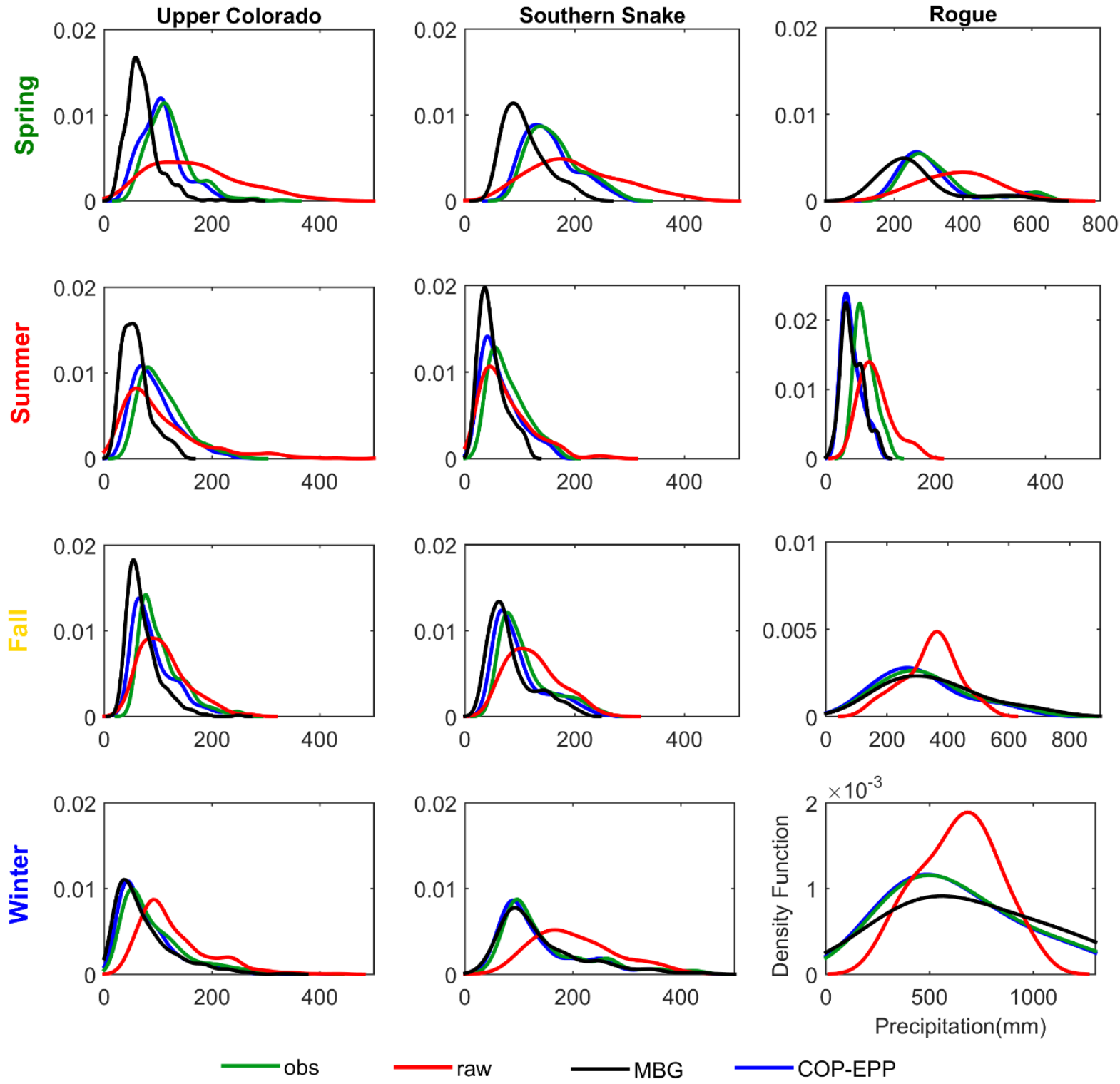

Figure 10. Probability density functions of seasonal precipitation from the observation, raw forecast, and most probable forecast based on the COP-EPP and MBG approach for three study basins. Seasonal precipitation is spatially averaged over all grid cells of each basin. Seasons are 
categorized in the following order: Spring (Mar, Apr, and May), summer (Jun, Jul, and Aug), fall (Sep, Oct, and Nov), and winter (Dec, Jan, and Feb). 
Table 1. Summary characteristics of the study basins

\begin{tabular}{|c|c|c|}
\hline Basin Name & Drainage Area $\left(\mathrm{km}^{2}\right)$ & Annual Precipitation $(\mathrm{mm})$ \\
\hline Upper Colorado & 280000 & 164 \\
\hline Southern Snake & 180000 & 493 \\
\hline Rogue & 5318 & 970 \\
\hline
\end{tabular}

